\section{Ringing the changes in chemistry}

\section{Robert Ramage}

Comprehensive Heterocyclic Chemistry:

The Structure, Reactions, Synthesis and Uses of Heterocyclic Compounds. Chairmen of the editorial board Alan R. Katritzky and Charles W. Rees. Pergamon: 1984. Eight volumes. £1375, \$2200.

HETEROCYCLIC chemistry is a crucially important area of organic chemistry; it embraces a wide range of structural types and applications; and the pharmaceutical industry is, to a large extent, sustained by developments in the structure, reactivity and synthesis of heterocyclic systems. Pergamon's eight-volume series has these three themes as the unifying factor, which has enabled the principal editors and the volume editors to achieve an internally consistent presentation embodying coverage of a remarkable range of topics. The necessary editorial control exercised in the compilation of 154 chapters has not stifled the independent scholarship of the many contributing authors - their collective efforts have resulted in a work which can truly be considered to be "comprehensive".

The series is subdivided into six parts, and the approach adopted by the editors can best be illustrated by the general individual chapters on structure, reactivity and synthesis in Vols 2, 4, 5 and 7. These contributions are not only interesting in themselves but are excellent introductions to the subsequent, more specialized material in each section.

The editors have used Vol. 1 (which constitutes Part 1) to deal with general matters such as nomenclature, review literature, biosynthesis and applications of heterocyclic compounds, thus allowing the following sections to deal more with fundamental principles. In addition, the first volume treats an assortment of lesser known heterocyclic systems which could not easily be assimilated into the accounts of the main heterocyclic systems. Chapter 1.03 on the review literature of heterocycles includes an impressive yet accessible collection of a wide range of references, subdivided into synthesis, reactions and properties, while biological applications are succinctly discussed in chapters dealing with biosynthesis, toxicity, and pharmaceutical, agrochemical and veterinary products. Chapter 1.17 by K. Dimroth, on heterocyclic rings containing phosphorus, is especially impressive in content and organization and will allow chemists to appreciate more fully this less traditional area of the subject.

Part 2 covers six-membered ring systems incorporating one nitrogen atom (Vol. 2) and then oxygen, sulphur, or two or more nitrogen atoms (Vol. 3). The treatment of these topics is consistent with the general editorial policy of logical development from structure through reactivity to synthesis. Highlights here are Chapter 2.03, a membered rings with varying degrees of unsaturation, and Chapters 2.05 and 2.06 which illustrate many facets of pyridine reVolume 2 will certainly provide good background reading in this general area, while the more specialized topics of Vol. 3 are treated in a clear, interesting manner. fundamental chemistry required for understanding of many of the biologically important heterocyclic systems.

In Vol. 4 (Part 3 of the series), the approach to five-membered rings containing one hetero-atom is in accord with the pattern set out in the previous volumes. The introductory section (Chapters 3.01, 3.02 and 3.03 ) has been authoritatively written by C.W. Bird and G.W.H. Cheeseman, and there are useful accounts of the application of spectroscopic methods to structural problems of five-membered heterocycles, resonance and tautomerism. Pyrroles and their benzo derivatives are dealt with in some detail, and are followed by an excellent chapter by K.M. Smith on porphyrins and related compounds which examines in depth the synthesis of simple corrinoids and vitamin $B_{12}$. The treatment of furan and thiophene chemistry is both a critical and an extensive contribution.

Both Vols 5 and 6 (Part 4) include accounts of a remarkable range of heterocycles containing two or more heteroatoms. Each class of ring system is treated consistently, and here are to be found strategic discussion of synthesis of sixactivity in monocyclic and bicyclic systems. Together, these two volumes contain the

heterocyclic types ranging from the biologically important imidazoles and purines to the more specifically chemically interesting systems of importance in organic synthesis.

Volume 7 (Part 5) is used to collate the less voluminous chemistry of heterocyclic systems having small and large rings. The treatment of small-ring chemistry is instructive and well written, although the coverage of $\beta$-lactam antibiotics is of necessity rather too brief to be of more than general interest. The chemistry of seven-membered and larger rings is dealt with clearly and concisely, and crown ethers and cryptands are thoroughly reviewed by A.D. Hamilton who takes the subject from consideration of crystal structures to biological and synthetic applications of coordination of these macrocycles containing several heteroatoms.

The final volume (and part) contains the index, helpfully divided into physical data, author, subject and ring. In addition, the work as a whole benefits from an ingenious reference code system which gives the reader easy access to original papers and source material such as books and reviews.

Comprehensive Heterocyclic Chemistry makes a unique contribution to the literature of organic chemistry. The references quoted are mainly post-1965, and together the eight volumes provide chemists, and biologists working at the chemical interface, with an impressive body of collected knowledge which is not available from any other source. Expensive though it is, chemistry libraries cannot afford to be without this series.

Robert Ramage is Forbes Professor of Organic Chemistry at the University of Edinburgh.

\section{Bacterial diplomacy}

\section{Howard C. Berg}

Motility and Taxis in Prokaryotes.

By A.N. Glagolev.

Harwood: 1984. Pp.279. \$126.

MODERN work on bacterial motility and taxis dates from the demonstration by Julius Adler (Science 166, 1588-1597; 1969) that the bacterium Escherichia coli has specific chemoreceptors: proteins near its outer surface that bind particular amino acids or sugars, causing the cell to respond favourably to changes in its environment, even in the absence of transport or metabolism. This meant that one could study behaviour without having to worry much about energetics, using genetic tricks to unravel steps in the sensory-transductive pathway.

In the Soviet Union, interest in this field was sparked by Vladimir Skulachev, well known for his work on chemiosmotic proton circuits, following the discovery by Larsen, Adler, Gargus and Hogg (Proc. natn. Acad. Sci. U.S.A. 71, 1239-1243;
1974) that bacterial flagella are powered, not by ATP, but rather by the high-energy intermediate of oxidative phosphorylation. Work in the Soviet Union, the United States and Japan has since shown that the immediate source of energy for flagellar rotation is, indeed, the protonmotive force. Alexei Glagolev, a protégé of Skulachev, now raises the Mitchellian banner and argues that $\Delta \bar{\mu} \mathrm{H}^{+}$plays an important role in tactic sensing and signalling.

This is a research monograph, not a book for the casual reader. It begins with a compact survey of bioenergetics, summarizes work on the motile mechanics of flagellated cells and gliders, and then presents an extensive discussion of various forms of directed movement, including chemo-, photo-, thermo-, visco- and magneto-taxis. The book brings together the results of a number of experiments described previously by the author and his colleagues in a series of short papers appearing primarily in FEBS Letters and FEMS Microbiology Letters.

While many of the phenomena described in these papers and reviewed here are striking and warrant serious attention - experiments on the behaviour of long trichomes 